\title{
Praxisnahe und effiziente ERP-Ausbildung am Beispiel der TU Ilmenau
}

\author{
Sebastian Büsch, Volker Nissen, Lutz, Schmidt \\ Fachgebiet Wirtschaftsinformatik für Dienstleistungen, \\ Technische Universität Ilmenau
}

\section{1 Überblick}

Mit dieser Arbeit werden Möglichkeiten aufgezeigt, eine universitäre Lehrveranstaltung so zu gestalten, dass sie einen hohen didaktischen, fachlichen und praktischen Anspruch erhält und den teilnehmenden Studenten mehr als nur ERP-spezifisches Fachwissen vermittelt. Um diesem Anspruch gerecht zu werden, wird die Lehrveranstaltung PROBAS an der TU Ilmenau als die universitäre Lehre ergänzendes SAP-Projekt organisiert. Der Beitrag zeigt auf, wie diese ERP-Ausbildung effizient aufgebaut, gemanagt und das Projektmanagement laufend verbessert wird, um kommenden Herausforderungen in der WI-Lehre zu begegnen. Es werden innovative Lehrkonzepte und fachliche Lehrinhalte an erfolgreichen, praktischen Beispielen vorgestellt, die sich im Zusammenhang mit einer universitären Ausbildung im ERP-Bereich über Jahre bewährt haben.

\section{Zweck und Zielstellung der Lehrveranstaltung}

PROBAS (Projektseminar betriebswirtschaftliche Anwendungssysteme) ist eine praxisorientierte Lehrveranstaltung der TU Ilmenau. Durch die langjährige Erfahrung bei der kontinuierlichen Betreuung der Lehrveranstaltung kann die Zielsetzung, bei der Ausbildung von Studenten an der TU Ilmenau, neben fachlichem ERP-Wissen auch weitere, praxisbezogene Fähigkeiten zu vermitteln, erreicht werden. Dazu zählen insbesondere das Entwickeln von Problemlösefähigkeiten, der Erwerb von Soft Skills und Erfahrungen im Projektmanagement sowie der Aufbau von Fähigkeiten zur Bewältigung realer Probleme. Das Erlernen von Kernkompetenzen im ERP-Bereich am Beispiel SAP sowie von fachbezogenem Wissen angrenzender Themen wie Business Intelligence (SAP-BI), Systemintegration (SAPPI) oder Anwendungsentwicklung (ABAP, SAP-EP) auf Basis der SAPNetweaver-Technologieplattform stehen ebenfalls im Mittelpunkt. 
Um den hohen Praxisbezug der vermittelten Inhalte zu erreichen, wird PROBAS als Projekt in Form einer praktischen Vertiefung theoretischer Inhalte angeboten und ergänzt darin bestehende ERP-Vorlesungen am Fachgebiet Wirtschaftsinformatik für Dienstleistungen der TU Ilmenau. Dadurch lässt sich ein in sich geschlossenes, aber weitestgehend selbstorganisierendes LehrLern-Arrangement verwirklichen. Durch den Einsatz eigener Funktionsträger im studentischen Projektteam, die für Bereiche des Wissens- und Marketingmanagements der Lehrveranstaltung zuständig sind, kann bei gleichbleibendem Betreuungsaufwand durch Lehrstuhl und Praxispartner eine kontinuierliche Weiterentwicklung des Projektes erfolgen. Insbesondere bei der Vorbereitung auf die Praxis durch Projektstruktur, Soft Skills und Problemlösefähigkeiten ergeben sich dadurch Vorteile gegenüber statisch-theoretischen Lehrveranstaltungen in Form von Vorlesungen. Durch die langjährige Erfahrung bei der Durchführung der Veranstaltung seitens des Lehrstuhls und der Praxispartner kann so ein geeignetes $\mathrm{Maß}$ an studentischem Engagement flexibel durch die Variation der Aufgabenstellungen oder den Einbezug der Studenten in die Projektorganisation erreicht werden.

Das Ziel dieses Beitrages ist es, Wege aufzuzeigen, ERP-Lehre so zu gestalten, dass sie einem universitären Anspruch genügt und gleichzeitig unmittelbar berufsrelevante Kenntnisse vermittelt. Es wird aufgezeigt, welche Konzepte wie in die Lehre mit welchen Vorteilen eingebunden werden können, welche Ergebnisse sich auf diese Art an praktischen Beispielen ergeben können oder welche Lehrformen und Lehrinhalte sich für eine SAP-Ausbildung anbieten. Dabei werden auch die Vorteile realer Problemstellungen aufgezeigt und dargelegt, wie durch praktische Aufgaben die Tauglichkeit der theoretischen Lehre für die Praxis evaluiert werden kann. Insbesondere das Zusammenspiel zwischen Universität und Praxis ermöglicht hierbei große Synergien, wodurch beide Seiten profitieren.

Im Rahmen dieses Beitrages wird auch dargestellt, wie durch die Verwendung quantitativer Maße zur Steuerung einer Lehrveranstaltung am Beispiel PROBAS ein effizient arbeitendes Projektmanagement etabliert werden kann. Mittels des CMMI-Reifegradmodells des Software Engineering Institute (2006) wird eine Methode der Evaluation der Lehrveranstaltung vorgestellt. Um eine Verbesserung der Qualität des Projektmanagements zu erreichen, schlagen wir eine Balanced Scorecard (BSC) nach Kaplan und Norton (1996) für das Projekt PROBAS vor.

\section{Verwendete Lehrmethoden}

Die angesprochenen Ziele einer fachlichen Wissensvermittlung auf der einen und die Vorbereitung auf das Lösen realer Probleme auf der anderen Seite erfordern ein detailliertes ERP-Verständnis seitens der Projektteilnehmer. Auf kognitiver Ebene haben sich die Lernziele nach Bloom (1956) etabliert. Das Ziel ist folglich der selbständige Weg des Nachvollzugs im Sinne eines „Wissen -> Verstehen -> Anwenden -> Analysieren -> Synthetisieren -> Evaluieren“. Während mit „klassi- 
schen“"Vorlesungen und Übungen das „Wissen und Verstehen“ (Vorlesung) sowie „Anwenden und Analysieren“ (Übung) abgedeckt werden kann, erfordern „Synthetisieren und Evaluieren“ weitergehende Lehrformen. Die Eignung von Fallstudien hierzu haben Funk et al. (2008, S. 1937 - 1947), Courant (2008, S. 1915 1926) und Freyburger und Lehmann (2008, S. 1927 - 1935) aufgezeigt. Um interessante Konzepte demonstrieren zu können, werden daher betriebswirtschaftliche Szenarien mit einem entsprechenden Datenbestand benötigt, die bei PROBAS durch das eigens erstellte und bearbeitete Modellszenario erreicht werden (siehe Kapitel 6). Der Einsatz von Fallstudien als Semesteraufgabenstellung ermöglicht ein realitätsnahes Arbeiten und einen entsprechend praxisnahen Wissensaufbau.

Die zur Bearbeitung notwendige Vorbereitung der Projektteilnehmer erfordert neben der fachlichen Wissensvermittlung insbesondere das Weitergeben impliziten Wissens zur eigenverantwortlichen Problembearbeitung. Für den Aufbau dieses Wissens erfolgt innerhalb von PROBAS der Einsatz von Coaching, Mentoring oder Job Rotation (Heinrich und Stelzer 2009, S. 446ff). Job Rotation wird in diesem Zusammenhang durch das Wechseln der Teilnehmer zwischen verschiedenen Aufgabenbereichen und Teilgruppen im Projekt erreicht.

Durch die Organisation der Lehrveranstaltung als Projekt kann neben dem Erlernen von Wissen und dessen Anwendung eine über die reine (aus universitärer Lehre heraus kritisch zu betrachtende) anbieterspezifische Wissensvermittlung der SAP-Kenntnisse hinaus ein breites Anwendungsgebiet erschlossen werden. Vor allem der Lösungsansatz einer Projektorganisation zur Förderung von Soft Skills entspricht einer modernen Didaktik; es wird nicht (nur) das reine Fachwissen präsentiert, sondern (auch) der Weg aufgezeigt, das Wissen eigenverantwortlich zu erlangen und auf andere (ERP-)Bereiche SAP-unabhängig zu übertragen. Die genaue Umsetzung der Lernmethoden in der praktischen Projektorganisation wird in den folgenden Kapiteln näher erläutert.

\section{Organisation der Lehrveranstaltung}

Das PROBAS-Projekt verfügt über eine hierarchische Aufbauorganisation mit Funktionsträgern aus den Bereichen Projektmanagement, Wissensmanagement und Marketingmanagement, sowie mehreren Arbeitsgruppen. Insgesamt besteht das Projektteam aus etwa 40 Studenten, die im Laufe der Semester kontinuierlich wechseln und in den meisten Fällen vier Gruppen zugeordnet sind. Die Projektstruktur für das Sommersemester 2009 zeigt Abbildung 1:

Der Projektleiter moderiert Workshops und Teamsitzungen als Projektverantwortlicher. Die übrigen Posten im Projektmanagement werden von Projektmitgliedern übernommen, die die Stellen eines zentralen Projektmanagers und die Teilprojektleiter als Ansprechpartner ihrer jeweiligen Gruppe besetzen. Der Teilprojektleiter ist direkt für die jeweilige Gruppe verantwortlich. Er überwacht die Erfüllung der Aufgaben und pflegt den Projektstatus zur Fortschrittskontrolle für 
das Projektmanagement. Der Projektmanager dient als zentraler Wissensmanager und übernimmt die Koordination und Kommunikation zwischen Teilprojektleitern und Projektleiter.

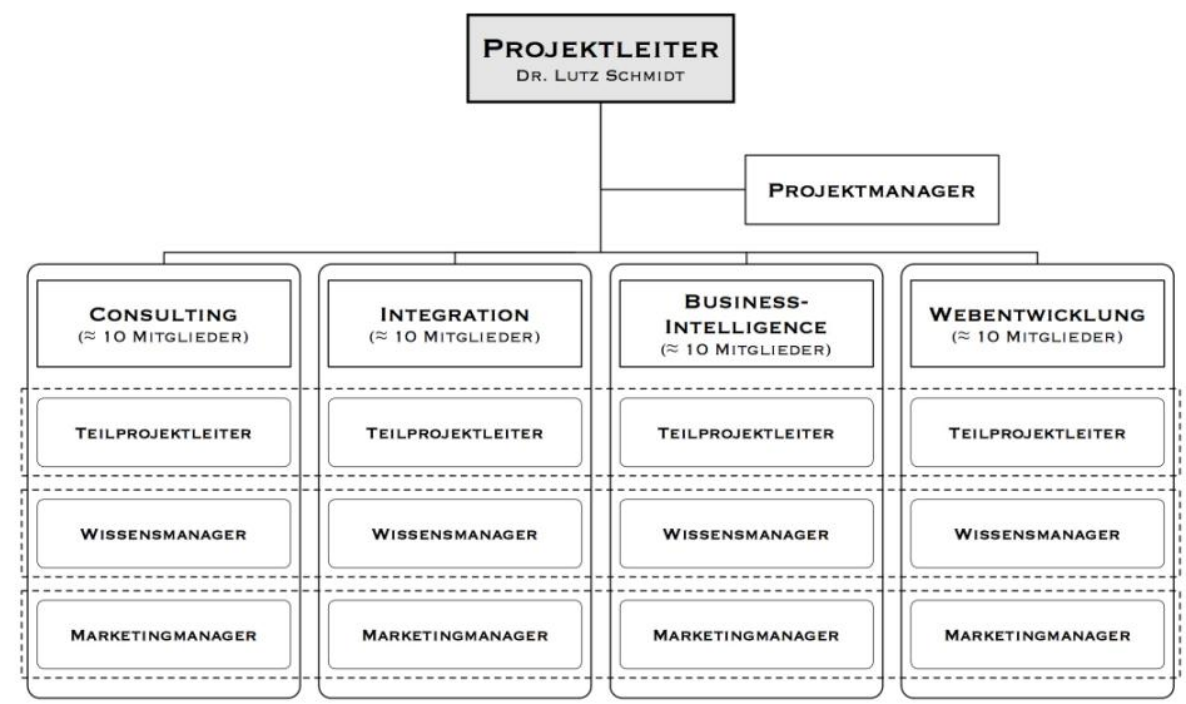

Abbildung 1: PROBAS-Projektstruktur

In jeder Gruppe wird außerdem ein Wissensmanager und Marketingmanager bestimmt. Die Hauptaufgabe der Wissensmanager ist es, Konzepte zur Verwaltung und Weitergabe des in der Lehrveranstaltung entstehenden Wissens zu entwickeln und durchzusetzen. Er leistet damit einen großen Beitrag zur Qualitätssicherung und zum Wissenstransfer innerhalb des Projektes. Die Marketingbeauftragten verbessern die Außendarstellung von PROBAS und informieren verschiedene Zielgruppen wie Studenten, Universitätsmitarbeiter und Unternehmen umfassend über das Projekt, beispielsweise durch die Organisation von Werbemaßnahmen und die Pflege der PROBAS-Homepage (www.PROBAS.de).

$\mathrm{Zu}$ Semesterbeginn werden die Inhalte der kommenden Veranstaltungsreihe festgelegt und die personelle Besetzung des Projektmanagements geplant. Das offizielle PROBAS-Semester beginnt mit einer Einschreibeveranstaltung, in der grundlegende Themen im ERP- und SAP-Bereich vermittelt und in deren Anschluss die Teilnehmer für das Semester ausgewählt und zu Arbeitsgruppen zusammengestellt werden. In den Gruppen werden Studenten mit jeweils unterschiedlichen Vorkenntnissen zusammengefasst. Neben Studenten, die erstmals am Projekt teilnehmen (Einsteiger), arbeiten in jeder Gruppe Studenten mit Erfahrungen aus vorangegangenen PROBAS-Semestern (Fortgeschrittene).

Nach der Einschreibeveranstaltung wird die inhaltliche Arbeit in einem Wochenend-Workshop als Blockveranstaltung fortgesetzt. Hier werden die fachlichen 
Grundkenntnisse vermittelt und erstmals gemeinsam in Gruppen Problemlösungen erarbeitet. Im weiteren Verlauf werden über das Semester die Aufgaben in den Arbeitsgruppen eigenständig bearbeitet und in zweiwöchentlichen Teamsitzungen der Ergebnisfortschritt präsentiert. Die Bearbeitungsphase endet mit dem zweiten Wochenend-Workshop. Das Projekt wird danach mit der Abschlussveranstaltung für das aktuelle Semester beendet, die die Ergebnisse des Projektes zusammenfasst und regelmäßig durch fachliche Vorträge von Unternehmensvertretern ergänzt wird.

\section{Erwerb und Weitergabe von Fachwissen und Soft Skills}

Die Organisation als Projekt erlaubt den Einsatz unterschiedlicher Formen der Wissensvermittlung. So erfolgt ein Zusammenspiel zwischen der Wissensvermittlung als Seminar, der Gruppenarbeit und dem Lernen durch Fehler und deren Bewältigung während der eigenständigen Aufgabenbearbeitung.

Basis der fachlichen Projektarbeit sind reguläre ERP-Vorlesungen und praktische Übungen zu Beginn des Projektes. Mittels Seminaren werden spezielle Lehrinhalte durch erfahrende Coaches aus der SAP-Beratungspraxis vermittelt. Im Laufe des Semesters geben zusätzlich Studenten Lehrinhalte in Vortragsform weiter, meist von Fortgeschrittenen zu Einsteigern. Zum Ende jedes Semesters werden im Rahmen eines Workshops relevante Themen von den Studenten erarbeitet und das erlernte Wissen zur abschließenden Aufgabenbearbeitung angewendet.

Zur Verwaltung der expliziten Wissensbasis wird ein eigenes Wissensmanagementtool (DHC-Vision) verwendet und durch die Wissensmanager verwaltet. ${ }^{1}$ Das PROBAS-Team nutzt und sammelt dokumentiertes Wissen in vielfältiger Form. Es entstehen Dokumente und Systemkonfigurationen. Persönlichen Erfahrungen und Nachschlagewerke werden gesammelt. Durch das zentrale Wissensmanagement wird diese Wissensbasis effektiv verwaltet und für spätere Semester zur Verfügung gestellt. Dadurch kann die Wissensbasis auch kontinuierlich erweitert und verbessert werden.

Eine stärker implizite Wissensvermittlung erfolgt durch die Aufgabenbearbeitung in Gruppen und den Coaching-Ansatz. Die Arbeitsgruppen setzen sich aus Studenten verschiedener Studienrichtungen von Informatik über Wirtschaftsinformatik bis zu Ingenieuren und Betriebswirten zusammen. Die Heterogenität wird verstärkt durch unterschiedliches theoretisches Vorwissen aus anderen Lehrveranstaltungen, teilweise vorhandene Praxiserfahrungen sowie Kenntnisse aus bereits absolvierten PROBAS-Projekten. Die heterogene Zusammensetzung der an gemeinsamen Aufgabenstellungen arbeitenden Gruppen fördert die Wissensweitergabe untereinander. Auch die Zusammenarbeit von Einsteigern, Fortgeschrittenen und Coaches ist hier ein wesentlicher Faktor. So geben Fortgeschrittene ihre Er-

\footnotetext{
${ }^{1}$ DHC-Vision (www.dhc-gmbh.com) dient der Ablage und Nutzung allen expliziten Wissens des Projektes und kann als spezielle Form eines Informationsmanagementsystems aufgefasst werden.
} 
fahrungen an Einsteiger weiter. Sehr erfahrene Studenten unterstützen außerdem als Coaches in Form von Vorträgen und direkt bei der Aufgabenbearbeitung. Auf diese Weise kann ein sehr schneller Wissensaufbau innerhalb des Projektteams erfolgen. Inhalt und Qualität der Wissensvermittlung wird durch den Projektleiter überwacht.

Das Vermitteln von Soft Skills, insbesondere Team- und Projektorganisationsfähigkeiten, innerhalb der Lehrveranstaltung ergibt sich aus der in sich geschlossenen Organisation von PROBAS. Es wird ein eigenes, für die Erreichung der Projektziele verantwortliches, Projektmanagement aus den Lehrverantwortlichen und Studenten gebildet. Das führt zu einer unmittelbar berufsverwertbaren Vorbereitung der Studenten auf projektorganisierte Aufgabenbewältigung in der betrieblichen Praxis. PROBAS deckt damit die aktuell am häufigsten gesetzten Zielstellungen in der Unternehmenssoftware-Ausbildung ab. ${ }^{2}$ Daneben werden aber auch weitere Bereiche der Wirtschaftsinformatik für die Praxis, wie IT-Beratung, Software-Entwicklung, Informationsmanagement etc. gefördert.

In ähnlicher Weise bereichern die Aufgaben der Wissensmanager und Marketingmanager sowohl das Projekt PROBAS an sich, als auch die Erfahrungen der studentischen Manager. Das Vorhandensein eines eigenen Marketingteams zur Gestaltung der PROBAS-Außenwirkung und die Selbstverwaltung des projekteigenen Wissensmanagements führen durch die damit verbundenen Aufgaben und Pflichten zu den entsprechenden Lernerfahrungen bei den ausführenden Studenten.

\section{Betrieb und Evaluation der Lehrveranstaltung auf Basis der PROBAS-Modellfabrik}

\subsection{Messung und kontinuierliche Verbesserung des PROBAS- Projektmanagements}

Im Rahmen einer kontinuierlichen Weiterentwicklung von PROBAS ist die Unterstützung des PROBAS-Managements durch eine Quantifizierung des Zielerreichungsgrades vorgesehen. Es soll eine bessere Bestimmung von Verbesserungspotentialen in der PROBAS-Lehre erreicht werden. Dazu ist es das Ziel, aus Sicht des Projektmanagements, Zielkriterien und Maße zur Bewertung des Projektfortschritts zu definieren, Ursache-Wirkungs-Beziehungen zwischen Maßnahmen und Zielerreichung zu bestimmen und Handlungsbedarf sowie Verbesserungsmaßnahmen während des Projekts abzuleiten.

Gemäß des Reifegradmodelles CMMI wird die Qualität des Projektmanagements in 5 Stufen (initial, managed, defined, quantitatively managed, optimizing)

\footnotetext{
2 Eine Studie des Lehrstuhls für Wirtschaftsinformatik der Technischen Universität München hat ergeben, dass die beiden Hauptausbildungsprofile in der SAP-Lehre eine Ausbildung zum SAPAnwender und Qualifizierung in Managementfragestellungen verfolgen (Schubert et al. 2008, S. 9).
} 
beschrieben. Für Stufe 2 (managed) werden die Kriterien „Existenz und Nutzung einer Prozessbeschreibung“, sowie die „Darstellung der Zielerreichung“ genannt. In Stufe 3 (defined) existiert darüber hinaus ein standardisiertes Vorgehen zur Projektabwicklung, das den Projektablauf komplett beschreibt und Entscheidungen für eintretende Situationen liefert. Anpassungen sind selten und situationsspezifisch nötig; es existiert ein kontinuierlicher Verbesserungsprozess, in den auch Erfahrungen einfließen.

Das aktuelle PROBAS-Projektmanagement wird wesentlich über einen vorhandenen Projektmanagementleitfaden gesteuert. Darin sind Hinweise für die Bereiche Projekt-, Wissens- oder Marketingmanagement, sowie der Projektablauf und Erfahrungswerte enthalten. Erfahrungen werden dokumentiert und weitergegeben. PROBAS wird kontinuierlich verbessert, indem Reviews durchgeführt werden und in den Leitfaden eingehen. Verbesserungen im Projektmanagement, das nahezu jedes Semester in seiner Besetzung wechselt, werden festgehalten. Ergebnisse werden überwacht, durch Meilensteine visualisiert und regelmäßig präsentiert (in Form von Statusvorträgen und in der Abschlusspräsentation). Aus Sicht des CMMI-Modells ergibt sich demnach ein PROBAS-Stand von Stufe 3 (defined), da ein kontinuierlicher Verbesserungsprozess etabliert ist und ein standardisiertes Vorgehen existiert.

Das zukünftige Ziel der PROBAS-Veranstaltung ist es, den Status 4 (quantitatively managed) zu erreichen. Dafür wird die Formulierung und Messung quantitativer Ziele gefordert. Ursache-Wirkungs-Beziehungen zwischen Maßnahmen und Zielen sind zu definieren. Aktuell gibt es bereits Ansätze für eine (auch quantitative) Messung des Projekterfolges. Z.B. werden die Anzahl der Bewerber oder die Teilnehmer der Abschlussveranstaltung erhoben und als Erfolgsmaß gewertet.

Weiterführende Maße und Ursache-Wirkungs-Beziehungen sollen im Kontext einer Balanced Scorecard definiert und verwaltet werden (siehe Kap. 6.3). Dadurch wird in Zukunft ein gezielter Einsatz leitender und überwachender Maßnahmen durch das Management erleichtert. Ist dieser Zustand erreicht, kann auch der höchste Reifegrad nach CMMI (optimizing) erreicht werden, der auf Basis der Beziehungen auf Stufe 4 alle relevanten Prozesse kontinuierlich verbessert und die Zielerreichung quantitativ überprüft. Eine Umsetzung ist durch den bereits vorhandenen kontinuierlichen Verbesserungsprozess sowie eine Ergänzung desselben um die identifizierten Ursache-Wirkungs-Beziehungen möglich.

Eine wesentliche Herausforderung in diesen Bemühungen stellt der ständig wechselnde „Personalbestand“ bei PROBAS dar. Dadurch wird die Weitergabe des Wissens und der Verantwortung zwischen den Studenten, die zumeist eine Verweildauer im Projekt von zwei bis vier Semestern aufweisen, erschwert. Dieses Problem wird durch den Wechsel der Studenten / Projektmitglieder aus den Diplomstudiengängen zu den Bachelor- und Masterstudiengängen verschärft. Oft wird nur noch sechs Semester studiert, was eine geringere Anzahl Semester, die sich die einzelnen Studenten bei PROBAS engagieren können, zur Folge hat. Die Studenten beginnen daher oft bereits im 1. bis 3. Semester und verfügen damit über we- 
niger Grundkenntnisse und Projektmanagementfähigkeiten als Studenten vergleichbarer Diplomstudiengänge. Die Wissensweitergabe und dessen Aufbau gestalten sich ebenfalls schwieriger. Die BSC stellt in diesem Kontext ein nützliches Hilfsmittel dar, um der komplexer gewordenen Personalsituation durch verbesserte Steuerung und Überwachung von Schlüsselzielen Rechnung zu tragen.

\subsection{Ausgewählte Teilprojekte}

\section{PROBAS-Modellfabrik}

Als Ausgangspunkt der PROBAS-Arbeit wird eine eigene Modellfabrik betrieben. In einem fiktiven Szenario werden betriebswirtschaftliche Prozesse abgearbeitet und im SAP-System abgebildet. Es werden dabei betriebliche Kernprozesse von Einkauf, Produktion und Vertrieb eines eigenen Produktes durchlaufen. Die Modellfabrik wird eigenständig von den Studenten durch Vertriebsvorgaben aus dem Projektmanagement betrieben. Dadurch entstehen produktive Bewegungs- und Stammdaten innerhalb des Systems durch dessen kontinuierliche, semesterübergreifende Nutzung. Diese Daten werden innerhalb des SAP-ERP-Systems für weitere Bereiche wie Rechnungswesen oder Controlling weiterverwendet. Die Arbeit im ERP-System erstreckt sich nicht auf das reine Abarbeiten von standardisierten Prozessen, sondern umfasst auch eigenes Customizing und die Weiterentwicklung des abgebildeten Szenarios, etwa durch Nutzung von Streckengeschäft oder Variantenkonfiguration. Der Ablauf der Modellprozesse wurde ergänzend mittels EPK dargestellt und weiterhin dokumentiert.

Fehler bei der Bearbeitung des Szenarios werden durch Fehlermeldungen festgehalten und für spätere Semester als Hilfe und Erfahrungswerte weitergegeben. Können Probleme dadurch nicht gelöst werden, wird an Fortgeschrittene, Coaches oder das Projektmanagement bei Bedarf eskaliert. Nicht für alle Fehler existiert eine eindeutige Fehlerbehebung und Vorgehensweise zur Lösung. Trotz dieses kontinuierlichen Verbesserungsprozesses über die Semester hinweg, kann heute noch nicht von einem Reifegrad der Stufe 4 (quantitatively managed) in diesem PROBAS-Bereich gesprochen werden.

\section{Netweaver-Fallstudie}

Durch das Vorhandensein realer und dynamisch erzeugter Daten aus einem praxisnahen Modellszenario können die Aufgaben der einzelnen PROBAS-Gruppen miteinander verbunden werden. Es werden SAP-Netweaver-Technologien verwendet, um - wie in der Unternehmenspraxis üblich - die eigenen Geschäftsprozesse effizienter abbilden zu können.

Durch Verwendung der SAP-PI zur Prozessintegration wird es ermöglicht, von beliebigen externen Systemen mit unterschiedlichen Schnittstellenkonfigurationen auf die Datenbestände der Modellfabrik zuzugreifen. Dadurch werden bei- 
spielsweise Business Partner oder Kundenaufträge durch PROBAS-externe Unternehmenspartner im System erzeugt.

Durch die Gruppe Webentwicklung wurde mittels Web Dynpro for ABAP eine Webumgebung zum Online-Vertrieb der PROBAS-Produkte entwickelt. Darin existiert auch ein Statistik-Bereich, der die Daten der Modellfabrik auswerten kann (implementiert mit Business-Graphics). Die komplette logistische Kette kann so per Webfrontend in einem deutlich reduzierten Zeitaufwand abgearbeitet werden, da die zeitintensiven und starren Dynpros der SAPGUI-Oberfläche in der Komplexität erheblich vereinfacht wurden.

Insbesondere der Bereich Business Intelligence bietet im Rahmen der PROBAS-Weiterentwicklung ebenfalls erhebliches Potential. So wurden die produktiven ERP-Daten der Modellfabrik an das SAP-BI-System angeschlossen und eine regelmäßige Aktualisierung implementiert. Das beinhaltet die Einrichtung des kompletten ETL-Prozesses, Prozessautomatisierung und Monitoring der Anwendung. Darauf aufbauend wurden Auswertungen und Cockpits ausgearbeitet, mit denen die Arbeit der Consulting-Gruppe verbessert werden kann, statt IDESTestdaten untersuchen zu müssen. Darin sind aggregierte, managementtaugliche Auswertungen sowie detailgenaue Untersuchungen möglich.

Die implementierten Cockpits und Datenbestände erlauben ein quantitatives Management und Bewerten der PROBAS-Fortschritte im Sinne eines Business Performance Management. Aktuell sind ein Monitoring der Ergebnisse der Modellfabrik sowie eine Fortschrittskontrolle durch verschiedene Kennzahlen möglich. Kennzahlen wie „Antwortzeit auf Kundenanfragen“, der „durchschnittliche Lagerbestand“" etc., die einen Rückschluss auf die Effizienz der Modellfabrik zulassen, können ausgewertet werden. Diese werden zukünftig auf die entwickelte BSC des folgenden Kapitels umgestellt und in bestehende Auswertungen flexibel integriert. Dadurch wird die Abfrage der Kennzahlen- und Dimensionsausprägungen der BSC möglich und damit auch die Messung und Steuerung der PROBASZielerreichung. Das Erreichen des Status „quantitatively managed“ lässt sich demnach mit den vorhandenen BI-Mitteln nach den definierten Ursache-WirkungsBeziehungen zukünftig umsetzen.

\subsection{Langfristige PROBAS-Steuerung mittels eigener Balanced Scorecard}

Aus den im Laufe des Beitrages beschriebenen Zielstellungen der Lehrveranstaltung lassen sich die PROBAS-Ziele in vier Dimensionen einer BSC abbilden, die in Abb. 2 dargestellt sind. Mittels dieser definierten BSC wird über die kommenden Semester das Projektmanagement gesteuert und in Bezug auf die effiziente Durchführung der Lehrveranstaltung weiter verbessert.

Zur Evaluation des Aufbaus von Fachwissen können mehrere Kriterien herangezogen werden. Es eignet sich insbesondere der Stand der Aufgabenerfüllung (gemessen an den Semesterzielen). Zusätzlich wird für Einsteiger bereits heute ein Wissenstest durchgeführt, um den Lernfortschritt zu kontrollieren. Die Er- 
folgsquote der Studenten in diesem Test liefert ebenfalls Rückschlüsse auf das Erlangen von Fachwissen. Als Kriterium wird außerdem die Nutzungshäufigkeit von Dokumenten (des Wissensmanagementsystems) eingesetzt.

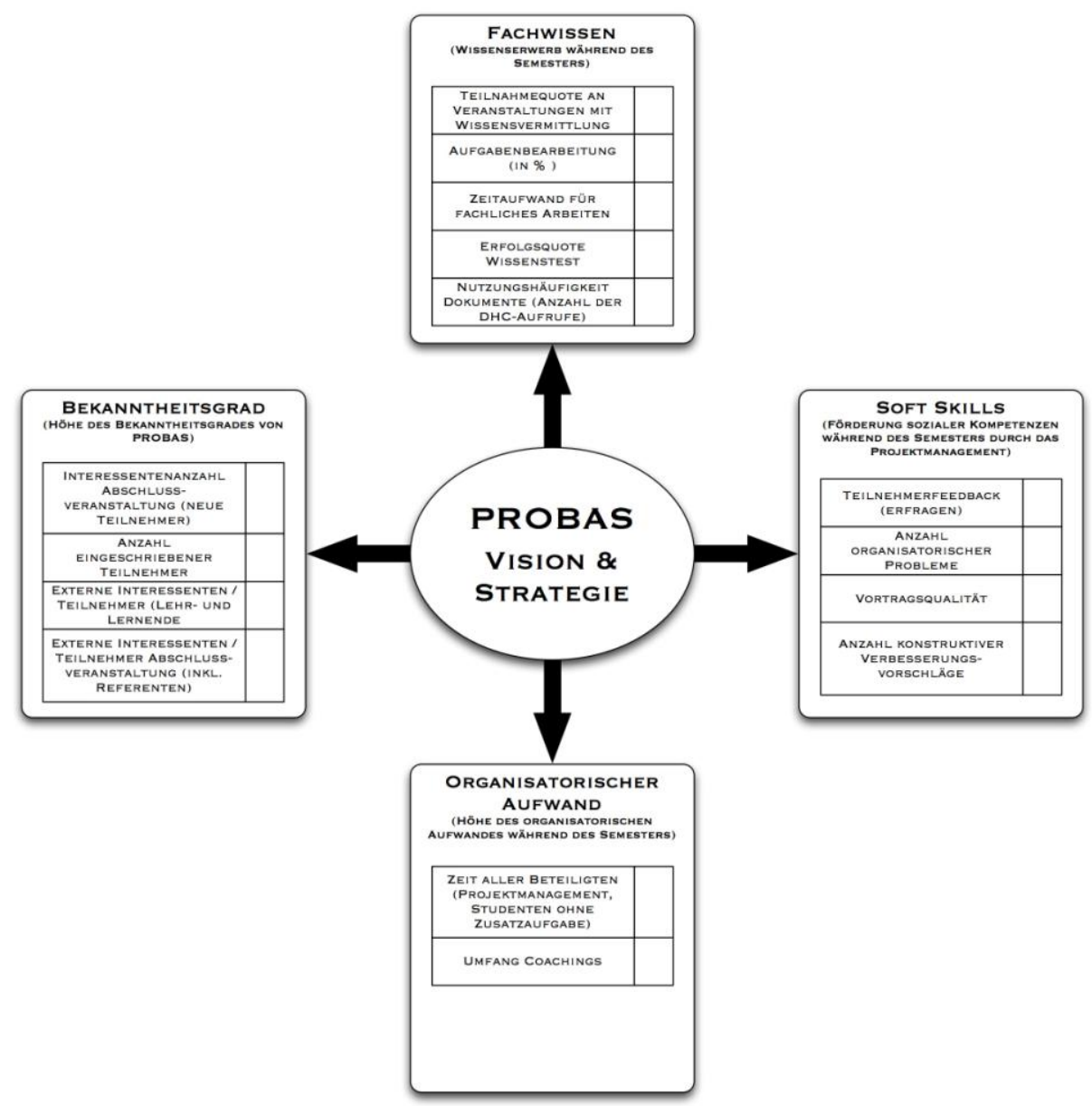

Abbildung 2: Balanced Scorecard zur Steuerung von PROBAS

Auch für die Vermittlung von Soft Skills können Kriterien gebildet und Daten erhoben werden. Die Ermittlung erfolgt vorrangig qualitativ durch Beurteilung der von Studenten gehaltenen Präsentationen im Rahmen von Statusmeetings, Fachvorträgen und Abschlussworkshops. Es wird ein Teilnehmerfeedback eingeholt und zur Verbesserung der Lehrveranstaltung berücksichtigt. Der Softskill Projektmanagement kann durch die Anzahl der erfassten und gelösten organisatorischen Probleme evaluiert werden, wobei proaktiv gelöste und reaktiv behandelte Prob- 
leme zu unterscheiden sind. Die Anzahl Verbesserungsvorschläge wird als Maß für Softskills im Bereich Ideenmanagement genutzt.

Ein möglichst hoher Bekanntheitsgrad der Veranstaltung kann anhand der Interessenten gemessen werden. Das betrifft die Anzahl der studentischen Interessenten und Bewerber für die Veranstaltung im Semester sowie deren Anwesenheit während der Abschlussveranstaltung. Insbesondere der Anteil von externen Interessenten aus der Praxis oder Studenten anderer Universitäten gibt Aufschluss über den Bekanntheitsgrad von PROBAS über die TU Ilmenau hinaus.

Zur Wahrung der Wirtschaftlichkeit des Projektes sollte auch der Aufwand zur Durchführung der Lehrveranstaltung bei gleichem Aufbau von Fachwissen und Soft Skills möglichst gering gehalten werden. Dies betrifft den organisatorischen Aufwand sowie den Bedarf an Coaching, der für den Aufbau eines spezifizierten Wissenstandes aufgewendet werden muss.

Mit den genannten Kriterien können wesentliche Anhaltspunkte für die Erreichung der Zielstellungen der Lehrveranstaltung ermittelt werden. Da die Ermittlung teilweise auf qualitativen Einschätzungen beruht und von unterschiedlichen Personen anhand subjektiver Wertbilder erfolgt, werden die Ergebnisse mit Unsicherheit behaftet bleiben. Es ist jedoch zu erwarten, dass trotz dieser Unsicherheiten deutliche Trends und Schwerpunkte erkennbar werden.

\section{$7 \quad$ Fazit}

PROBAS als Projektseminar betriebswirtschaftliche Anwendungssysteme ist eine praxisorientierte Lehrveranstaltung der TU Ilmenau mit dem Ziel, durch eine projektartige Organisation Studenten effizient den Umgang mit SAP-Systemen näher $\mathrm{zu}$ bringen. Es wird jedoch nicht nur Fachwissen vermittelt, sondern auch Soft Skills entwickelt. Durch die Verknüpfung fachlicher Aufgabenbereiche mit der Organisation eines Projekts werden Fähigkeiten wie Organisieren, Koordinieren, Delegieren oder Kommunizieren gefördert und eine gute Zusammenarbeit der Gruppen untereinander ermöglicht.

PROBAS ist eine Lehrveranstaltung, die in hohem Maße von studentischer Mitarbeit abhängig ist und entsprechendes Engagement fördert. Die Motivation der Teilnehmer ist generell hoch. Mittels PROBAS kann an der TU Ilmenau ein breites Methodenspektrum in der WI vermittelt werden. PROBAS ergänzt die theoretisches Wissen vermittelnden universitären ERP-Vorlesungen durch ein Angebot, das praktisch interessierten Studenten eine Vertiefungsmöglichkeit bietet, die auch für die beruflichen Chancen nach dem Studienende bedeutsam ist.

Das Beispiel PROBAS zeigt, wie sich verschiedene Lernformen einer SAPAusbildung ergänzend umsetzen lassen. Die Ergebnisse haben einen hohen theoretischen, praktischen und technologischen Anspruch vor dem Hintergrund praxisnaher Problemstellungen in der eigenen Modellfabrik. PROBAS zeigt auch, wie 
Vertreter der Unternehmenspraxis und universitäre Lehre zusammenarbeiten und voneinander profitieren können.

\section{Literatur}

Bloom B (1956) Taxonomy of Educational Objectives, Handbook I: The Cognitive Domain. McKay, New York.

Courant J (2008) Beispiele für den Einsatz von SAP-Software in der Wirtschaftsinformatikausbildung im Kontext eines Lernmodells. In: Bichler K, Hess T, Krcmar H, Lechner U, Matthes F, Picot A, Speitkamp B, Wolf P (Hrsg.): Multikonferenz Wirtschaftsinformatik 2008, Berlin.

Freyburger K, Lehmann P (2008) Herausforderungen bei der

Wirtschaftsinformatik-Ausbildung mit Standardsoftware am Beispiel von mySAP Business Intelligence. In: Bichler K, Hess T, Krcmar H, Lechner U, Matthes F, Picot A, Speitkamp B, Wolf P (Hrsg.): Multikonferenz Wirtschaftsinformatik 2008, Berlin.

Funk B, Niemeyer P, Rautenstrauch C, Scheruhn HJ, Weidner S (2008) Modellierung und Implementierung eines Order2Cash Prozesses in verteilten Systemen. In: Bichler K, Hess T, Krcmar H, Lechner U, Matthes F, Picot A, Speitkamp B, Wolf P (Hrsg.): Multikonferenz Wirtschaftsinformatik 2008, Berlin.

Heinrich LJ, Stelzer D (2009) Informationsmanagement - Grundlagen, Aufgaben, Methoden. Oldenbourg, München.

Kaplan RS, Norton DP (1996) The Balanced Scorecard: Translating Strategy Into Action. Harvard, Boston.

Schubert U, Wittges H, Krcmar H, Schrader H (2008) UnternehmenssoftwareAusbildung: Aktueller Stand und Trends. Ergebnisse der 5. University Alliances Bildungsbedarfsanalyse 2008. Arbeitspapier Nr. 14, Garching.

Software Engineering Institute (2006) CMMI for Development, Version 1.2. Pittsburgh. 